\title{
Fronto-Normal Gait Incorporating Accurate Practical Looming Compensation
}

\author{
${ }^{*}$ Tracey K. M. Lee, ${ }^{1}$ M. Belkhatir, ${ }^{2}$ P.A. Lee and ${ }^{\dagger}$ S. Sanei \\ *School of EEE, Singapore Polytechnic, 500 Dover Road, S(139651) \\ ${ }^{*}, 1,2$ School of IT, Monash University Sunway Campus, Jalan Lagoon Selatan, 46150 Petaling Jaya \\ ${ }^{\top}$ Centre of Digital Signal Processing, Cardiff University, Cardiff, CF24 3AA. \\ *tlee@sp.edu.sg, ${ }^{1}$ Belkhatir.Mohammed@infotech.monash.edu, ${ }^{2}$ lee.poh.aun@infotech.monash.edu.my ${ }^{\dagger}$ saneis@cf.ac.uk
}

\begin{abstract}
Gait is an emerging biometric showing promise in its use. Most research focusses on fronto-parallel $(F P)$ gait where people walk across a camera. In this paper, we present an original analysis, presenting the case for the use of frontonormal (FN) gait where motion is towards a camera. In FN gait, the image projected on a camera sensor will get larger, in a looming effect. This affects the data in a nonlinear and non stationary way, which will further complicate analysis of movement. By catering for this effect, we present new insights into perspective motion compensation for FN gait. Using an existing database which uses coloured markers, we compare two methods of compensation for looming. Initial examination of the resulting data shows a significant result, that fundamentally different approaches may be used for time series analyses on the a set of FN gait data. This opens up new avenues for biometric research in gait recognition.
\end{abstract}

\section{Introduction}

As a biometric, gait has desirable properties. It can be used at long distances, is non-intrusive, non-invasive, and is hard to disguise. In the literature, the main gait recognition approaches analyse walking which proceeds in a plane parallel to a camera, the so-called fronto-parallel (FP) view. This gives the largest variation in a silhouette from which time series data is obtained for analysis. From a far distance, this is advantageous.

Motion from a plane perpendicular to this, the frontonormal view (FN), is considered as a special case. However we present a case for $\mathrm{FN}$ gait to be considered in its own right as a biometric. In Section 2, we present the case for FN gait analysis. Section 3 covers our setup and Section 4 considers some geometric factors in analyzing FN gait. Section 5 show some results with preliminary analysis and we conclude with Section 6.

\section{Overview of Gait Analysis}

In this section, we provide an overview of human recognition using gait which concentrates on providing the motivation for FN gait. In the literature, the area of gait analysis and recognition has involved medical analyses looking for exact movement of body parts to detect pathological conditions. However, various types of sensors have to be attached to the body to do this. In security applications this is not useful. The uses of gait as a biometric for human identification have been covered in works such as [1]. Much of the current gait analyses use silhouettes in the FP view because of the large changes in shape. In contrast, applications like computer animation use 3D body modelling to analyse generic human motion. This is too computationally intensive and we consider a simplified $2 \mathrm{D}$ view of a human performing a repetitive activity i.e. walking, which is more tractable.

\subsection{Space constraints}

Commonly, people are made to queue up to access a facility. In a corridor like structure, we assume that a subject is approaching a camera. Depending on the type of analysis need, in a FP walk, at least two cycles or four steps are needed. For more robust estimation of the period of walking, about $8 \mathrm{~m}$ is recommended [2]. To capture this movement, the camera distance required is about $9 \mathrm{~m} \mathrm{[3]} \mathrm{This} \mathrm{is} \mathrm{because}$ current video cameras typically have a focal length and sensor size of $8 \mathrm{~mm}$ and $1 / 2$ " respectively. Practically, having such a wide uncluttered space is difficult, since whenever we want to measure a person's gait, many people and objects will be present.

In a FN view, we can still use the $8 \mathrm{~m}$. But this time, we cover twelve steps and we only need a corridor-like structure, the width being about that of a human body. Therefore, considerable amount of space is saved as shown in Fig 2 in this case, by 2/9.

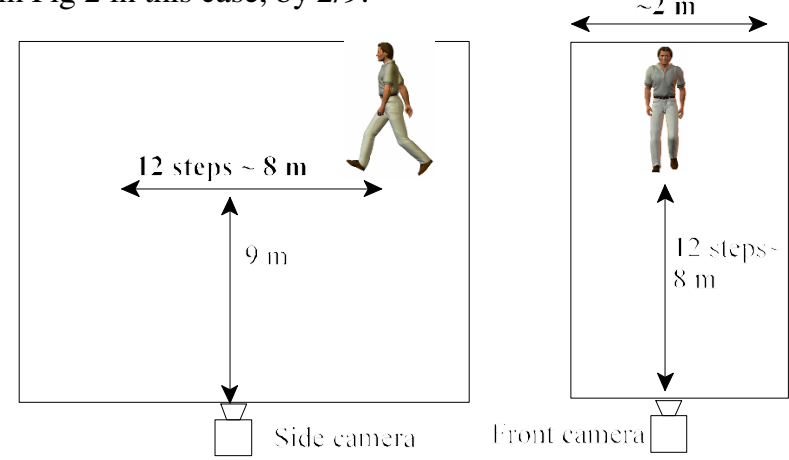

Fig. 1. FP vs FP - physical dimensions for video capture

\subsection{Combination of biometrics}

Several combinations of biometrics have been tried. For example, face and speech, face and iris and so on. Face with gait has been relatively unexplored, and these have used mainly the FP approach [4][5]. However, in using this approach, Zhou and Bhanu [6] use a profile view of a face with gait in order to use one camera at $3.3 \mathrm{~m}$ from the subject. The work by Bazin [7] includes the ear and footfall as biometrics. In most cases, they need two cameras where problems of alignment and synchronisation are significant. A single camera or monocular capture of video is preferred. 
Table 1 Types of biometric combinations possible with the two views of gait in a monocular set up

\begin{tabular}{|c|l|l|}
\hline Biometric & \multicolumn{1}{|c|}{ FP (side view) } & \multicolumn{1}{c|}{ FN } \\
\hline Face & Not reliable & $\begin{array}{l}\text { Frontal - well } \\
\text { researched }\end{array}$ \\
\hline Gait & $\begin{array}{l}\text { Good segmentation } \\
\text { strong periodicity }\end{array}$ & $\begin{array}{l}\text { Difficult to process } \\
\text { Can use nonlinear }\end{array}$ \\
\hline Iris & Not possible & Near distance use \\
\hline Ear & Not sure of usefulness & dubious use \\
\hline
\end{tabular}

From Table 1, we see that the FN view allows one to use face, iris and gait for a robust recognition system.

\subsection{Multiple observations}

As a follow on of the requirement for a smaller physical area, the FN view allows us to easily monitor the gait of several people at one time.
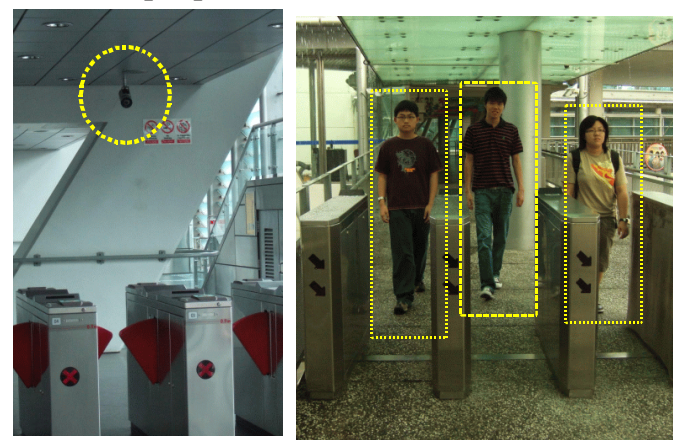

Fig. 2. Left, view of typical security camera monitoring access point. Right, tracking multiple subjects

However for the FP view, occlusion among subjects is common.

\subsection{Psychophysical and other experiments}

The research by Wang et al. [8] show that even FN silhouettes give better recognition performance.

Troje [9] has shown that the task of recognizing gender from Moving Light Displays has a better error rate using the FN view. This corresponds to earlier works by gait researchers as mentioned in his paper.

\subsection{Dynamic information}

In earlier papers, Lee et al. [10] show that FN gait can be characterised by nonlinear measures which show potential to be used as a biometric. Also, a variety of time series analyses may be employed further to characterise the gait such as in [11]. In contrast, FP gait yields mainly periodic measures.

\subsection{FN gait advantages}

The main advantages of monocular FN non-silhouette approach are:

i) Smaller physical space is needed.

ii) Multiple subjects can be tracked.

iii) Other biometrics can be easily combined.

iv) Wide variety of time analysis including non-periodic motion analysis can be used.

However, the FN view is challenging, having to compensate for the looming effect.

\section{Experimental Environment}

For gait recognition, we use feature points that have more motion in the image plane. This would be the hands, feet and knees, for a FP walk. For a FN walk this is also true, although the motions are smaller in magnitude. For the two kinds of walk, we use coloured markers set up as shown in Fig. 3.

The marker designations are: $l h / r h$ - left/right hand : $l f / r f$ - left/right foot : $l k / r k$ - left/right knee. Two additional discs of the same colour are attached at the waist and face level. They can be used for normalization, both of scale and distance due to the looming effect of a FN walk. They are: $\mathrm{tm} / \mathrm{bm}$, the top/bottom markers. The markers are tracked using the CAMSHIFT[12] algorithm. We take video clips of twelve subjects and from three of these, a second clip for testing.

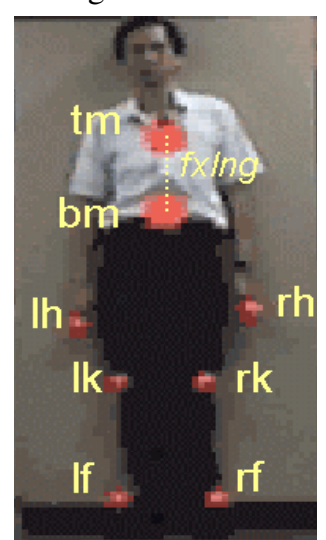

Fig. 3. FN Marker designations

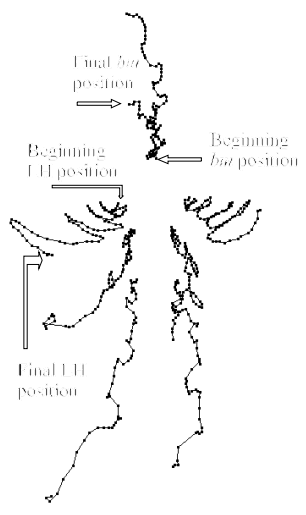

Fig. 4. Trajectories of marker for FN gait
Fig. 4 shows the trajectories of markers as a person walks toward the camera.

\section{Geometrical Considerations of FN Gait}

In the field of clinical gait, precise terms are used for the body structures and movements used in gait. We use the nomenclature introduced by Perry [13] in describing the phases in gait. As in all image based gait analysis, the projection of the $X$ and $Y$ coordinates on the sensor plane, $x$ and $y$. We follow the approach of Trucco and Verri[14] in relating the various image coordinate systems. In Eqn. (1) $\mathbf{P}^{\mathrm{c}}$ and $\mathbf{P}^{\mathrm{w}}$ are the vectors representing the camera and world coordinates of a point $\mathbf{P}$. $\mathbf{R}$ is the rotation matrix and $\mathbf{T}$ the translation vector used to convert between these two coordinate systems. Note the superscripts ${ }^{c}$ and ${ }^{w}$ are also used in other equations.

$$
\mathbf{P}^{c}=\mathrm{R} \mathbf{P}^{\mathrm{w}}+\mathbf{T}
$$


The camera equations between the image and camera plane, the coordinates on the sensor, $x$ and $y$ are given by:

$$
x=\frac{f}{Z^{c}} X^{c}+o_{x} \quad \text { and } \quad y=\frac{f}{Z^{c}} Y^{c}+o_{y}
$$

where $f$ is the focal length, $o_{x}$ and $o_{y}$ the offset of the image from the sensor origin. $Z^{C}$, is the object distance from the camera lens as shown in Fig 6. By expanding Eqn. (2) and using the elements of matrix $\mathbf{R}$ and vector $\mathbf{T}$, we have:

$$
\begin{aligned}
& x-o_{x}=-f_{x} \frac{r_{11} X^{w}+r_{12} Y^{w}+r_{13} Z^{w}+T_{x}}{r_{31} X^{w}+r_{32} Y^{w}+r_{33} Z^{w}+T_{z}} \\
& y-o_{y}=-f_{y} \frac{r_{21} X^{w}+r_{22} Y^{w}+r_{23} Z^{w}+T_{y}}{r_{31} X^{w}+r_{32} Y^{w}+r_{33} Z^{w}+T_{z}}
\end{aligned}
$$

To simplify this, we fix the axes of the human walker to be parallel to the camera so there is no rotation. Then $r_{11}, r_{22}$ and $r_{33}$ are 1 , and the rest will be zero including $\mathbf{T}$. Since we are only interested in the movement of the markers, we can use the change in distance between previous marker positions. Using a constant $K$ for invariant quantities, we have:

$$
\delta x=K \frac{\delta X^{w}}{Z^{w}} \quad \text { and } \quad \delta y=K \frac{\delta Y^{w}}{Z^{w}}
$$

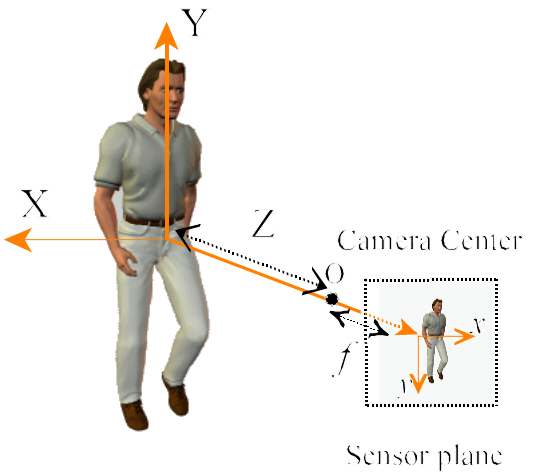

Fig. 5. Subject and projection on the camera plane (rotated $x-y$ axes)

Thus a challenging aspect of FN gait is the looming effect. This causes movements of the human body in the camera plane to become nonlinear and nonstationary due to the inversely changing value of $Z^{w}$. Of course, this is not a problem for FP gait as the human movement is essentially in one fixed plane. In fact, papers on biometrics which discuss the looming problem only compensate for the angle of walk [15]. Given our setup, we simplify notation and use $Z$ for $Z^{w}$ for the remaining discussion.

\subsection{Normalization Considerations}

In this paper, we look at two ways to normalize the coordinates in what we call motion based and dimension based normalization. The first approach uses the motion of the walker to estimate the $Z$ distance and from there, use projection equations. The second uses the dimensions of body parts only.
4.1.1 Dimension based Normalization. This has been used in [10], but to recapitulate, we normalize for scale by using the distance $f x \ln g$ between the $\mathrm{tm}$ and $\mathrm{bm}$ markers as seen in Fig 3. Secondly, we use one of the markers, $b m$ as the origin of movement. No depth information is used, making it more general to use as no prior human model is assumed.

4.1.2 Motion based Normalization. Another way of compensating for the looming effect is to use the projective equations shown in Eqn. (2). We consider the person walking in the $Z$ direction at a constant speed. This may give us smoother trajectories for the markers by generating a model for the movement. A model allows us to predict the motion of the coloured marker when total occlusion happens in FN gait motion.

To do so, we need to compute i) the length of limbs and ii) frequency of limb movement and from this, the speed of walking. Because of the irregular movements of the $t m$ and $\mathrm{bm}$ markers, as seen in Fig. 4, the motion of the other markers have a more irregular motion as well. There is also a linear trend in the data caused by the walking path.

\section{Length of limbs}

Approximating the motion of limbs with a single pendulum should provide satisfactory results. Since we do not know the length of the flexed arm used in walking, we estimate the available anthropometric data found in the image and extrapolate from there, using commonly found ratios, for example between arm length and height, found in standard anthropometric texts such as that found in Tilley et al.[16] and ratios in various biomechanical texts [2][13].

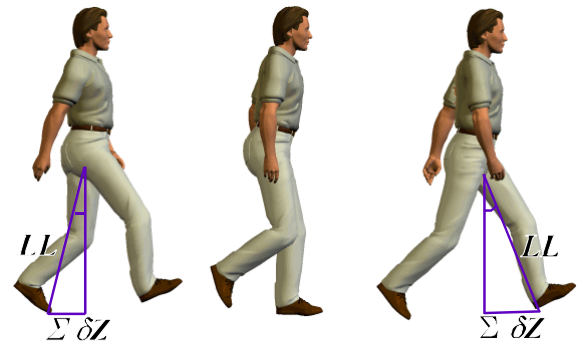

Fig. 6. Arm positions for a) Initial contact b) Load contact c) Terminal stance of walking

Frequency of limb movements

The frequency is computed by using the time periods between the inflection points of the gait data. In this case we base the motion of the body using the leg swing, using periodic assumptions, in the manner of of Unuma et al. [17]. They use a frequency domain approach to simulate human motion. Assuming a small angle of swing, with $L L$ the leg length, $f_{i}$ the frequency of motion at a given sample $i$, the subject moves $\delta Z_{i}$ in the z-axis in one sampling period $t_{s}$ in Eqn. (5), where $k$ is an adjustment factor. If the original subject-camera distance is $Z_{\text {org }}$, the expression for the distance $Z$ over $t$ samples is given by Eqn. (5)

$$
\begin{gathered}
\delta Z_{i}=k L L\left(2 \pi f_{i}\right) t_{s} \\
Z=Z_{\text {org }}-\sum_{1}^{t} \delta Z_{i}
\end{gathered}
$$

Due to space constraints, we show the results for a single subject. The results are similar for the rest of the subjects. 


\section{Results}

In Figs. 7 and 8 we show respectively, the plots of motion based and dimension based normalized gait data. As a final step, we also linearly detrend the motion based normalization data to compensate for the linear motion of the markers.

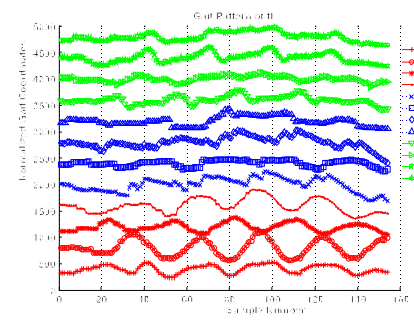

Fig. 7. Motion-normalized plot

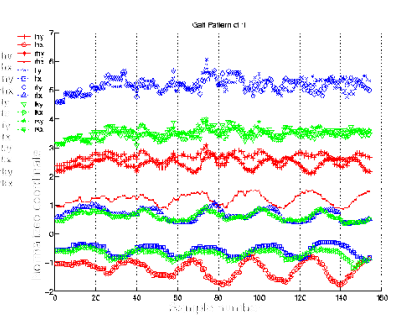

Fig. 8. Dimensionnormalized plot
Next, we perform an the autocorrelation analysis of the time series data for both types of normalization.

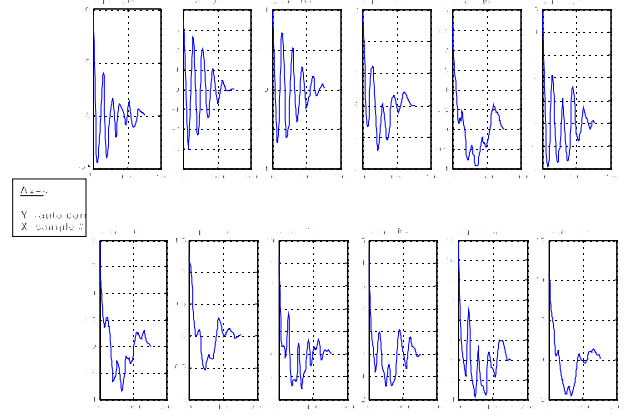

Fig. 9. Autocorrelation plot of Motion based normalized data

Fig. 9 shows oscillatory behaviour in the motion of the markers, raising the possibility of using linear time series analysis on the data.
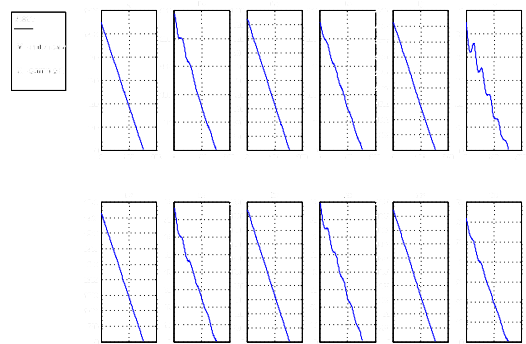

Fig. 10. Autocorrelation plot of Dimension based normalized data

As mentioned in earlier work [10], there is no sign of oscillatory behaviour in Fig. 10, indicating a need for nonlinear analysis. Table 2 compares the differences between the two types of normalization.

Table 2 Comparison of the normalization types

\begin{tabular}{|c|l|l|}
\hline Feature & \multicolumn{1}{|c|}{ Motion based } & \multicolumn{1}{c|}{ Dimension based } \\
\hline Model & Assumes a model & No model assumed \\
\hline Occlusion & Compensate & Can't handle \\
\hline Analyses & Linear types & Nonlinear types \\
\hline
\end{tabular}

\section{Conclusions}

In this paper, we furnished some compelling reasons for further investigation into $\mathrm{FN}$ gait as a natural platform for human identification. In adjusting for FN movement, we provide fresh insights into how we can effectively normalize for such movement without using extensive calculations. Finally, the autocorrelation plots indicate a linear type of time series analyses can be employed for motion based normalized data whereas nonlinear types would be used in dimension based normalization. In [10] we successfully incorporated nonlinear gait characteristics to enhance a face recognition system. Future work will compare the effectiveness of both types of normalized data in biometric applications. Finally, we acknowledge the useful comments by the anonymous reviewers which improved the paper.

\section{REFERENCES}

1. T.K.M.Lee, S. Ranganath and S. Sanei, "Patterns in paces: a survey of current approaches in gait recognition," Proc. Asian Biometric Workshop, pp 421-425, Singapore, Nov 2003. 2. M. W. Whittle, Gait Analysis: an Introduction, $4^{\text {th }}$ ed., Philapdelphia: Butterworth-Heinemann, 2007, pg 139.

3. G. Verhoeven, "Did the Digital (R)Evolution Change the Concept of Focal Length?" AARGNEWS, vol. 34, pp. 30-35 Mar 2007.

4. G. Shakhnarovich, L.Lee and T. Darrel, "Integrated Face and Gait Recognition with Multiple Views," CVPR, Kaui, HI, Dec 2001.

5. A. Kale, A. K. RoyChowdhury and R. Chellappa, "Fusion of Gait and Face for Human Identification," ICASSP, Montreal, Canada, May 2004.

6. X. Zhou and B. Bhanu, "Feature Fusion for Face and Gait for Human Recognition in Video," ICPR, Hong Kong, Aug 2006.

7. A. I. Bazin, On Probabilistic Methods for Object Description and Classification," PhD Thesis, University of Southampton, UK, 2006

8. L. Wang, T. Tan, "Silhouette Analysis-Based Gait Recognition for Human Identification," IEEE Trans. PAMI, vol.25, no.12, pp.1505 - 1518 , Dec 2003.

9. N. F. Troje, "Decomposing biological motion: A framework for analysis and synthesis of human gait patterns.," Journal of Vision, vol.2, pp.371-387, 2002.

10. T. K. M. Lee, S. Ranganath and S. Sanei, "Frontal viewbased gate identifaction incorporating the largest Lyapunov exponents," ICASSP, Toulouse, France, May 2006.

11. T.K.M. Lee, K.F. Loe, P.A. Lee and S. Sanei, "A comparison of the basic temporal features of Fronto-Normal and FrontoParallel Gait," DSP, Cardiff, UK, Jul 2007.

12. G. R. Bradski, "Computer video face tracking for use in a perceptual user interface," Intel Technology Journal, vol.Q2, 1998.

13. J. Perry, Gait Analysis: Normal and Pathological Function, First edition, Slack Incorporated, 1992.

14. E. Trucco and A. Verri, Introductory Techniques for 3-D Computer Vision, Upper Saddle River, NJ, Prentice Hall, 1998.

15. A. Kale, A.K Roy-Chowdhury and R. Chellappa, "Towards a View Invariant Gait Recognition Algorithm," AVSS, Miami, FL, Jul 2003.

16. A.R. Tilley and Henry Dreyfuss Associates, The Measure of Man and Woman: Human Factors in Design, Revised, New York, Wiley, 2002.

17. M. Unuma, K, Anjyo and R. Takeuchi, "Fourier principles for emotion-based human figure animation," Proc. SIGGRAPH ‘95, Los Angeles, CA, Aug 1995. 